\title{
Monitoring respiratory infections in covid-19 epidemics
}

Ka Hung Chan and colleagues argue that monitoring influenza-like illness could be a complementary approach to assessing the effectiveness of general infection control measures against covid-19

\author{
Ka Hung Chan early career research fellow ${ }^{1}$, Pak-wing Lee masters student ${ }^{2}$, Crystal Ying Chan \\ doctoral student ${ }^{3}$, Kin Bong Hubert Lam associate professor ${ }^{1}$, Pak-leung Ho clinical associate \\ professor $^{4}$
}

${ }^{1}$ Clinical Trial Service Unit and Epidemiological Studies Unit, Nuffield Department of Population Health, University of Oxford, Oxford, UK; ${ }^{2} \mathrm{Faculty}$ of Epidemiology and Population Health, London School of Hygiene and Tropical Medicine, London, UK; ${ }^{3}$ JC School of Public Health and Primary Care, Chinese University of Hong Kong, Hong Kong SAR, China; ${ }^{4}$ Carol Yu Centre for Infection and Department of Microbiology, University of Hong Kong, Hong Kong SAR, China

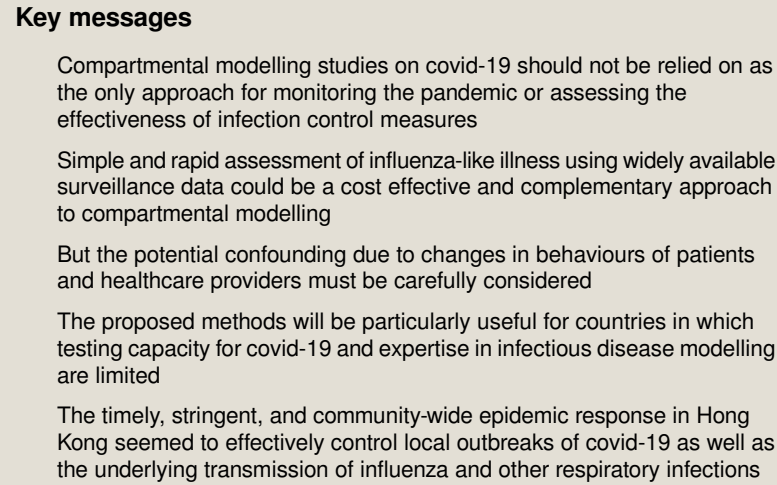

Compartmental modelling studies on covid-19 should not be relied on as the only approach for monitoring the pandemic or assessing the effectiveness of infection control measures

Simple and rapid assessment of influenza-like illness using widely available surveillance data could be a cost effective and complementary approach to compartmental modelling

But the potential confounding due to changes in behaviours of patients and healthcare providers must be carefully considered

The proposed methods will be particularly useful for countries in which testing capacity for covid-19 and expertise in infectious disease modelling are limited

The timely, stringent, and community-wide epidemic response in Hong Kong seemed to effectively control local outbreaks of covid-19 as well as the underlying transmission of influenza and other respiratory infections

The covid-19 epidemic has grown rapidly from a local outbreak in China to a pandemic with over 2.5 million confirmed cases and 166000 deaths worldwide (as at 20 April 2020). ${ }^{1}$ Despite the strong connection with mainland China, the number of confirmed cases in Hong Kong Special Administrative Region has been relatively low $(n=1025 ; 13.8$ per 100000 population as of 20 April, compared with 1651 per 100000 in New York City and 214.7 per 100000 in London), ${ }^{1}$ perhaps attributable to its extensive infection control response.

Limitations of compartmental modelling studies and the severe shortfall of covid-19 testing capacity worldwide, especially in low and middle income countries, have necessitated alternative options to appraise the performance of infection control strategies. Common respiratory infections (such as influenza) share similar transmission pathways (primarily through droplets and fomites) with covid- $19,{ }^{2}$ so most infection control measures should have qualitatively similar effects on these diseases, even though covid-19 is inherently more contagious. ${ }^{34}$ Examining reliable influenza surveillance data therefore offers a simple approach to indirectly assess the overall effectiveness of a population's general infection control strategies.

\section{Unique challenges in understanding covid-19 epidemiology}

Compartmental modelling has been crucial in understanding the covid-19 pandemic, estimating both the current scale ("nowcasting") and its development over time ("forecasting") to inform the epidemic response worldwide. But many of the assumptions made (such as probability of transmission per contact) are based on expert opinion because empirical evidence is often not available. ${ }^{5}$ Thus, a small error can be amplified into a large bias that can be consequential. The model that was used to inform the 2001 foot and mouth disease epidemic, which led to the culling of 1.2 million British livestock, was later found to have markedly overestimated the disease impact and underestimated the effectiveness of conventional public health intervention. $^{6}$

Modelling for covid-19 faces several unique challenges. First, the proportion of undetected, mild, or asymptomatic cases, which might account for a substantial proportion of community transmission, remains largely uncertain. ${ }^{3}$ Second, epidemic curves from different populations are not comparable and do not necessarily approximate the underlying transmission dynamics. Third, the fatality rate of covid- 19 varies dramatically from about $1 \%$ in Germany to $12 \%$ in Italy, warranting extra caution when inferring the actual number of infected people based on the number of deaths and fatality rates. ${ }^{1}$

\section{A simple, rapid, and cost effective approach}

Influenza (or influenza-like illness (ILI), defined by the World Health Organization as an acute respiratory illness with a 
measured temperature of $\geq 38^{\circ} \mathrm{C}$ and cough, with onset within the past 10 days) has been routinely monitored in 114 countries across five continents, coordinated by the Global Influenza Surveillance and Response System since 1952. ${ }^{7}$ Although high income countries tend to have stronger influenza surveillance, over the past few decades robust electronic surveillance systems have been established in Africa (13 countries), Latin America (23 countries), and the Middle East (14 countries). ${ }^{7}$

By monitoring the transmission patterns of ILI, we can approximate the overall performance of a population's general infection control measures, including enhancement of personal and environmental hygiene and social distancing. Other measures that are specific to covid-19, such as travel restrictions, quarantine, and case isolation, are unlikely to alter local ILI patterns substantially. Unlike covid-19, which has a long incubation period of up to 16 days, ${ }^{8}$ ILIs (incubation period 1-2 days $)^{9}$ are likely to be more sensitive to infection control measures. This allows a much more timely understanding of the changing transmission intensity of droplet and fomite borne disease, which includes covid-19.

Drastic reductions in various viral respiratory infections were also noted in Hong Kong during the SARS epidemic in 2003. ${ }^{10}$ Nonetheless, changes in healthcare seeking behaviours and disease surveillance practices, especially during stringent "lockdown" measures, must be carefully considered. The influence of behaviour and practice changes could be accounted for by examining ILI detection rates (which account for changes in the total number of tests or cases examined) and severe cases, deaths, or institutional outbreaks that are less likely to be confounded.

\section{The case of Hong Kong}

The covid-19 and ILI statistics from Hong Kong since January 2020 provide some evidence that ILI surveillance can provide information about the impact of general infection control measures.

The key infection control strategies undertaken in Hong Kong against covid-19 are case identification and isolation, quarantine, social distancing, and mass masking, all of which aim to reduce the contact rate between the infected and susceptible populations and the probability of transmission per contact (box 1). The epidemic response in Hong Kong is characterised by timeliness and strong civilian commitment-for example, mass masking (75-99\% prevalence of frequent mask use in public spaces) was achieved shortly after the first cases arrived. ${ }^{4}$

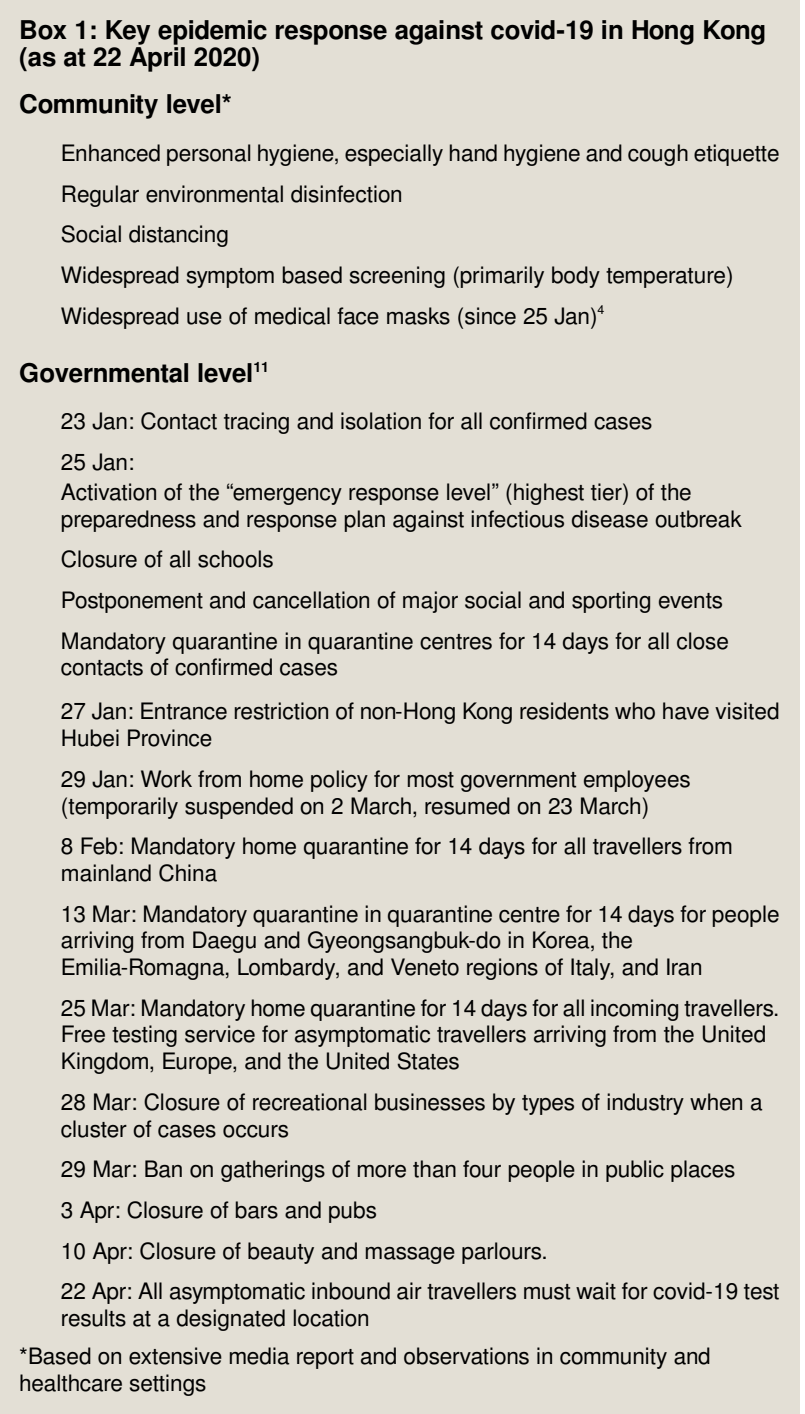

Hong Kong has had two "waves" of covid-19 outbreaks, the first occurring in weeks 6-11 of 2020 (134 cases, 32.1\% imported) and the second wave is ongoing since week 11 (875 cases as of 18 April, $63.1 \%$ imported) after a major influx of residents returning from epidemic struck regions worldwide (fig 1).

\section{Epidemiology of influenza and other respiratory infections}

Using published surveillance data, we compared the length and severity of the 2019-20 winter influenza season in Hong Kong with those in 2015-19. ${ }^{12}$ An influenza season in Hong Kong is the period in which the positive rate of influenza cases in respiratory tract specimens tested in the public health laboratory and the hospital admission rate for influenza are above the usual baseline $(9.2 \%$ for specimens positive rate and 2.5 per 100000 population for hospital admission rate; estimated as 1.96 standard deviation above the average weekly numbers during non-season periods from 2014 week 49 to 2019 week 48). ${ }^{12} \mathrm{We}$ also compared the positive test rates of common respiratory infections in Hong Kong before and after week 5 of 2020, when most infection control measures began.

Compared to 2015-19, the 2019-20 winter influenza season was $63.2 \%$ shorter, the number of institutional (schools or homes for elderly people) ILI outbreaks was $68.4 \%$ lower, and the number of deaths from laboratory confirmed influenza in adults 
was $62.3 \%$ lower (table 1). Although the 2020 season began at a similar time (week 2) as the previous winter (week 1), there was a sharp decline in laboratory confirmed influenza cases from $>18 \%$ in weeks $3-4$ to $<1 \%$ in week 8 and remained low $(0.1 \%)$ into week 15 , while the total number of respiratory specimens tested remained steady ( 6000 a week) (fig 2 , table 1). Similar trends have been observed for other respiratory infections in the same period (fig 2).

Consistently, the outpatient ILI rate (number of ILI cases per 1000 consultations in the sentinel general outpatient clinics) in Hong Kong started to fall after the first covid-19 case in week 4 , and this trend continued with a $>80 \%$ reduction between weeks 2 and 15 (fig 1).

We also looked at temporal ILI patterns (using population specific parameters) in Southern China (Hubei and 14 other provinces or municipalities, which recorded $92 \%$ of all reported covid-19 cases in China as at 18 April), South Korea, Singapore, and England. ${ }^{12-16}$ We found similar rapid reductions of ILI transmission from week 4 in Southern China (fig 3) and South Korea (fig 4) and from week 5 in Singapore (fig 5). ILI transmission intensity in Singapore started to rise from week 8 to week 11, and then dropped again until week 15 , which is similar to the trend observed in England over the same period (fig 6). Note that the ILI and covid-19 data across populations were only qualitatively compared because of substantial heterogeneity in the choice of indicator for ILI transmission and case identification strategies for covid-19 in different populations. For the same reasons, different scales were used for the $y$ axes of the figures.

\section{Interpretation}

The markedly shorter and less severe influenza epidemic and the rapid decline in incidence of common respiratory infections in Hong Kong coincided with the country's measures against covid-19. Although Hong Kong is now experiencing a second wave of covid-19 outbreak, primarily driven by imported cases from Europe and the US, local transmission in the second wave remains limited, with only 18 local covid-19 cases in weeks 15 and 16. As the number of returning residents starts to decline, the rate of expansion of the second wave seems to be falling. This is in line with the persistently low intensity of ILI transmission, indicating that the sustained local infection control measures might have hampered the chain of local transmissions of respiratory infections in Hong Kong.

Southern China, South Korea, and Singapore have also undertaken similarly rapid epidemic response measures since early February and have seen declines in ILI transmission in their first wave of covid-19 outbreaks. Unlike Hong Kong, South Korea experienced a major outbreak in weeks 9-10, primarily driven by several specific "superspreading" clusters (5016 cases in the Shincheonji Church cluster as of 18 March). ${ }^{17}$ Otherwise, the sustained low transmission of ILI is consistent with success in curbing the spread of covid-19. On the other hand, both Singapore and England have experienced a four week surge of ILI transmission in weeks 8-11, whereas the covid-19 cases reported in both populations started to increase rapidly from around week 11.

Like Hong Kong, Singapore has been experiencing a second wave of covid-19 driven by imported cases (70\% of all cases from 20 March to 2 April).$^{18}$ Both cities have been relatively successful in identifying and isolating imported cases, ${ }^{19}$ but they both experienced a resurgence of ILI at the end of the first wave of covid-19, and Singapore had a more severe second wave, potentially attributed to increased mobility in public or at workplaces and lower prevalence of mask use. ${ }^{1920}$

The gap of around three weeks between the rise of ILI and covid-19 in Singapore and England seems to coincide with the 2-3 week lag between community transmission and case identification of covid-19, due to the long incubation period and delays in testing and reporting. ${ }^{21}$ Furthermore, declining ILI transmission in Singapore and England in weeks 11-13 corresponds with the heightened infection control response. In particular, the mobility index in England, which had been stable in weeks $1-11$ of 2020 , dropped dramatically after news of the government's "herd immunity" plan (week 11). ${ }^{20}$ Although effectiveness of the response actions cannot be assessed yet, the changes in ILI transmission seem to offer some promising hints for England.

\section{Limitations}

Two major concerns for the proposed approach are that during a pandemic, as for most non-urgent illnesses, people with ILI might stop (or be told to stop) attending clinics, and the number of tests ordered for influenza surveillance might also be reduced. In such circumstances, however, the total consultation counts (the denominator of outpatient ILI rate) also decrease. The covid-19 tests in many countries might, at least partly, compensate for the changes, as people with covid-19 (non-specific respiratory) symptoms who test negative are documented as having ILI. A simple syndromic surveillance system for self-reported covid-19 or ILI symptoms (or both) could augment the proposed approach, with the advantages of being low cost and adaptive as it requires no additional clinical staff or laboratory capacity and could capture a more complete picture of covid-19 and ILI transmission in the community even with changing behaviours in patient and healthcare providers during the epidemic.

Importantly, the ILI data across populations cannot be quantitatively compared due to the substantial heterogeneity in the choice of indicator for ILI transmission, the virology of the specific strains, and vaccination efficiency and uptake rate. The same is true for covid-19, given the widely different case identification systems across populations. The drop in ILI GP consultation rate in England occurred before the local covid-19 outbreak and might not be relevant to this analysis. Overall, the ecological nature of our analysis prevents us from inferring causality or disentangling the independent effect of specific measures, and individual level analysis is needed for further investigation.

\section{Lesson learnt}

From the populations examined, we have shown that the transmission intensity of ILI was qualitatively consistent with that of covid-19, showing the potential value of the well established ILI surveillance data. Variations in the second wave in Hong Kong, primarily driven by imported cases, serve as a negative control showing how the approach might tease out the potential effects of general infection control measures. The rapid changes in ILI transmission support our argument that ILI is likely to be more sensitive to infection control measures than covid-19. The analysis of ILI might enable rapid appraisal of the impact of control measures on the transmission of covid-19 without mass serology testing.

The indirect evaluation method capitalising on the more widely available and reliable influenza surveillance data could be a complementary and cost effective way to understand different 
countries' performance in controlling covid-19, especially for low and middle income countries with limited resources. Syndromic surveillance would be a valuable addition to the existing Global Influenza Surveillance and Response System and for countries not yet included in this system. We have shown the feasibility of using a simple analytical framework with minimal demand on statistical expertise in populations with little testing capacity for covid-19. We suggest the use of nowcasting and time series analyses for enhancing the value of ILI monitoring during this pandemic.

Contributors and sources: All authors conceived the study together; PL and CYC collated and analysed the data; PL and KHC prepared the first draft of the report; $\mathrm{KHC}$ supervised $\mathrm{PL}$ and $\mathrm{CYC}$, and $\mathrm{KBHL}$ and $\mathrm{PH}$ oversaw the conduct of the study as senior authors; all authors contributed to the subsequent revision and final approval of the present manuscript. $\mathrm{KHC}, \mathrm{PL}$, and CYC contributed equally to this manuscript. $\mathrm{KBHL}$ and $\mathrm{PH}$ are the senior authors. $\mathrm{KHC}$ is the guarantor of the paper. All data analysed are publicly available and the sources are referenced. We thank colleagues at the Public Health Research Collaborative (PHRC) and Brian Leung at vote4.hk in Hong Kong Special Administrative Region of China for their contributions in data collection and graphics production. We also thank reviewers Ben Cowling and Pia Hardelid for their useful comments to improve the manuscript.

Patient involvement: The present manuscript involved two non-academic members of the PHRC, who have limited professional knowledge about the topic of interest. They reviewed the initial drafts of the article and provided comments to simplify the writing to better suit the general audience of The BMJ.

This work is not funded. KHC is a recipient of the NDPH Early Career Research Fellowship from the University of Oxford.

Competing interests: No conflict of interest is declared.

1 Dong E, Du H, Gardner L. An interactive web-based dashboard to track COVID-19 in real time. Lancet Infect Dis 2020;S1473-3099(20)30120-1.

10.1016/S1473-3099(20)30120-1 32087114

2 World Health Organization. Coronavirus disease 2019 (covid-19): situation report 73.2 Apr 2020. https://www.who.int/docs/default-source/coronaviruse/situation-reports/ 20200402-sitrep-73-covid-19.pdf?sfvrsn=5ae25bc7_6
3 Ferretti L, Wymant C, Kendall M, etal . Quantifying SARS-CoV-2 transmission suggests epidemic control with digital contact tracing. Science 2020;eabb6936.

10.1126/science.abb6936 32234805

4 Cowling BJ, Ali ST, Ng TWY, etal . Impact assessment of non-pharmaceutical interventions against coronavirus disease 2019 and influenza in Hong Kong: an observational study. Lancet Public Health 2020;S2468-2667(20)30090-6.32311320

5 Ferguson N, Laydon D, Nedjati Gilani G, et al. Report 9: impact of non-pharmaceutical interventions (NPIs) to reduce covid-19 mortality and healthcare demand. Imperial College COVID-19 Response Team. 16 Mar 2020. https://www.imperial.ac.uk/media/imperialcollege/medicine/sph/ide/gida-fellowships/Imperial-College-COVID19-NPI-modelling-1603-2020.pdf

6 Mansley LM, Donaldson Al, Thrusfield MV, Honhold N. Destructive tension: mathematics versus experience--the progress and control of the 2001 foot and mouth disease epidemic in Great Britain. Rev Sci Tech 2011;30:483-98. 10.20506/rst.30.2.2054 21961220

7 World Health Organization. Global influenza surveillance and response system (GISRS). https://www.who.int/influenza/gisrs_laboratory/en/.

8 Lauer SA, Grantz KH, Bi Q, etal . The incubation period of coronavirus disease 2019 (COVID-19) from publicly reported confirmed cases: estimation and application. Ann Intern Med 2020. 10.7326/M20-0504 32150748

9 World Health Organization. Influenza (seasonal). https://www.who.int/news-room/factsheets/detail/influenza-(seasonal).

10 Lo JY, Tsang TH, Leung YH, Yeung EY, Wu T, Lim WW. Respiratory infections during SARS outbreak, Hong Kong, 2003. Emerg Infect Dis 2005;11:1738-41. 10.3201/eid1111.050729 16318726

11 Hong Kong SAR Government. Covid-19 thematic website. https://www.coronavirus.gov hk/eng/index.html.

12 Centre for Health Protection. Flu express. 2020. https://www.chp.gov.hk/en/resources/ 29/304.html.

13 Chinese National Influenza Centre. Weekly influenza report. 18 Apr 2020 http://www. chinaivdc.cn/cnic/zyzx/lgzb/.

14 KCDC. Korea influenza weekly report. 17 Apr 2020. https://www.cdc.go.kr/board/board. es? $\mathrm{mid}=\mathrm{a} 30504000000 \& \mathrm{bid}=0033$.

15 Singapore Ministry of Health. Weekly infectious diseases bulletin. 8 Apr 2020. https:// www.moh.gov.sg/resources-statistics/infectious-disease-statistics/2020.

16 Public Health England. Weekly national flu reports: 2019 to 2020 season. 16 Apr 2020. https://www.gov.uk/government/statistics/weekly-national-flu-reports-2019-to-2020-season.

17 Reuters. The Korean clusters-how coronavirus cases exploded in South Korean churches and hospitals. 20 Mar 2020. https://graphics.reuters.com/CHINA-HEALTH-SOUTHKOREACLUSTERS/0100B5G33SB/index.html.

18 Singapore Ministry of Health. Daily report on covid-19. https://www.moh.gov.sg/covid-19/ situation-report.

19 Lewis R, Yap JCH. Covid-19: the response in Singapore. https://www.nuffieldtrust.org uk/news-item/covid-19-the-response-in-singapore 9 Mar 2020

20 Google. Covid-19 community mobility reports. https://www.google.com/covid19/mobility/

21 Wu Z, McGoogan JM. Characteristics of and important lessons from the coronavirus disease 2019 (COVID-19) outbreak in China: summary of a report of 72314 cases from the Chinese Center for Disease Control and Prevention. JAMA 2020 (in press) 10.1001/jama.2020.2648 32091533

Published by the BMJ Publishing Group Limited. For permission to use (where not already granted under a licence) please go to http://group.bmj.com/group/rights-licensing/ permissions 


\section{Table}

Table 1/ Comparing the Hong Kong winter influenza season in 2020 with those in 2015 to $2019^{9}$

\begin{tabular}{|c|c|c|c|c|c|}
\hline Winter influenza season & Week of the year & Length (weeks) & $\begin{array}{c}\text { Number of institutional } \\
\text { outbreaks* }\end{array}$ & Number of deaths & $\begin{array}{c}\text { Mean number of specimens tested } \\
\text { per week }\end{array}$ \\
\hline 2015 & 1 to 17 & 17 & 433 & 502 & 3908 \\
\hline 2016 & 5 to 21 & 17 & 435 & 214 & 4298 \\
\hline 2017 & 8 to 15 & 8 & 87 & 42 & 4039 \\
\hline 2018 & 2 to 13 & 12 & 600 & 384 & 6697 \\
\hline 2019 & 1 to 14 & 14 & 863 & 357 & 6486 \\
\hline 2015-2019, mean (SD) & NA & $13.6(2.5)$ & $483.6(191.7)$ & $299.8(107.0)$ & $5086(1384)$ \\
\hline 2020 & 2 to 7 & 5 & 153 & 113 & 6203 \\
\hline
\end{tabular}




\section{Figures}

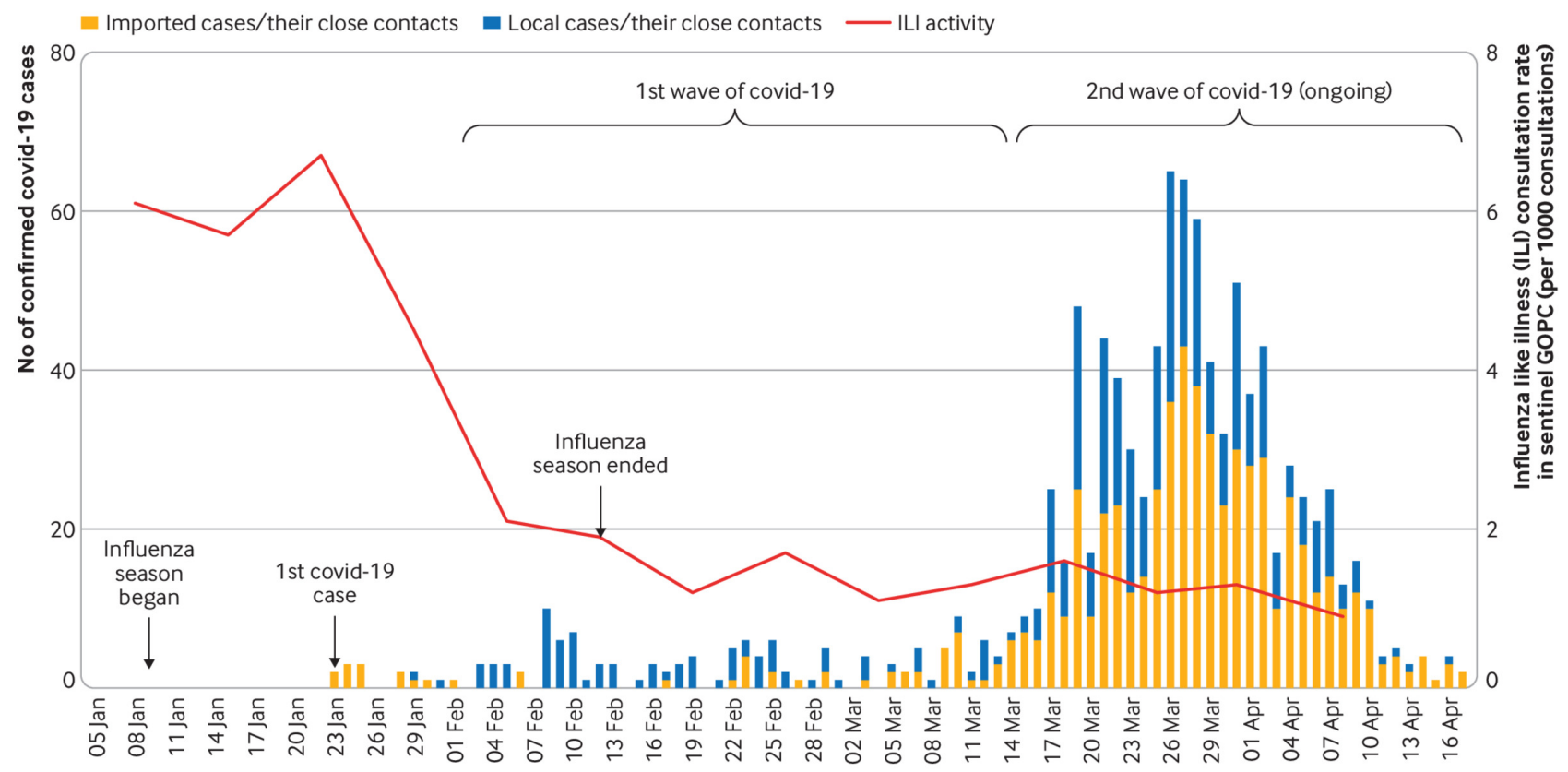

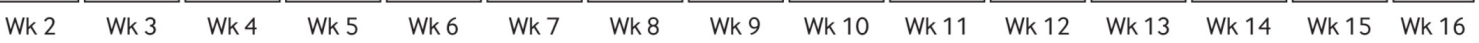

Fig 1 Incidence of covid-19 and indicators of transmission intensity of influenza-like illness from week 2 to 16 of 2020 in Hong Kong. Influenza season is defined as the period in which the positive rate of influenza cases in respiratory tract specimens tested in the public health laboratory and the hospital admission rate for influenza is above the usual baseline level. GOPC=public general outpatient clinics. Data source: Centre for Health Protection. ${ }^{12}$ The latest data available at the time of writing were dated 18 April.

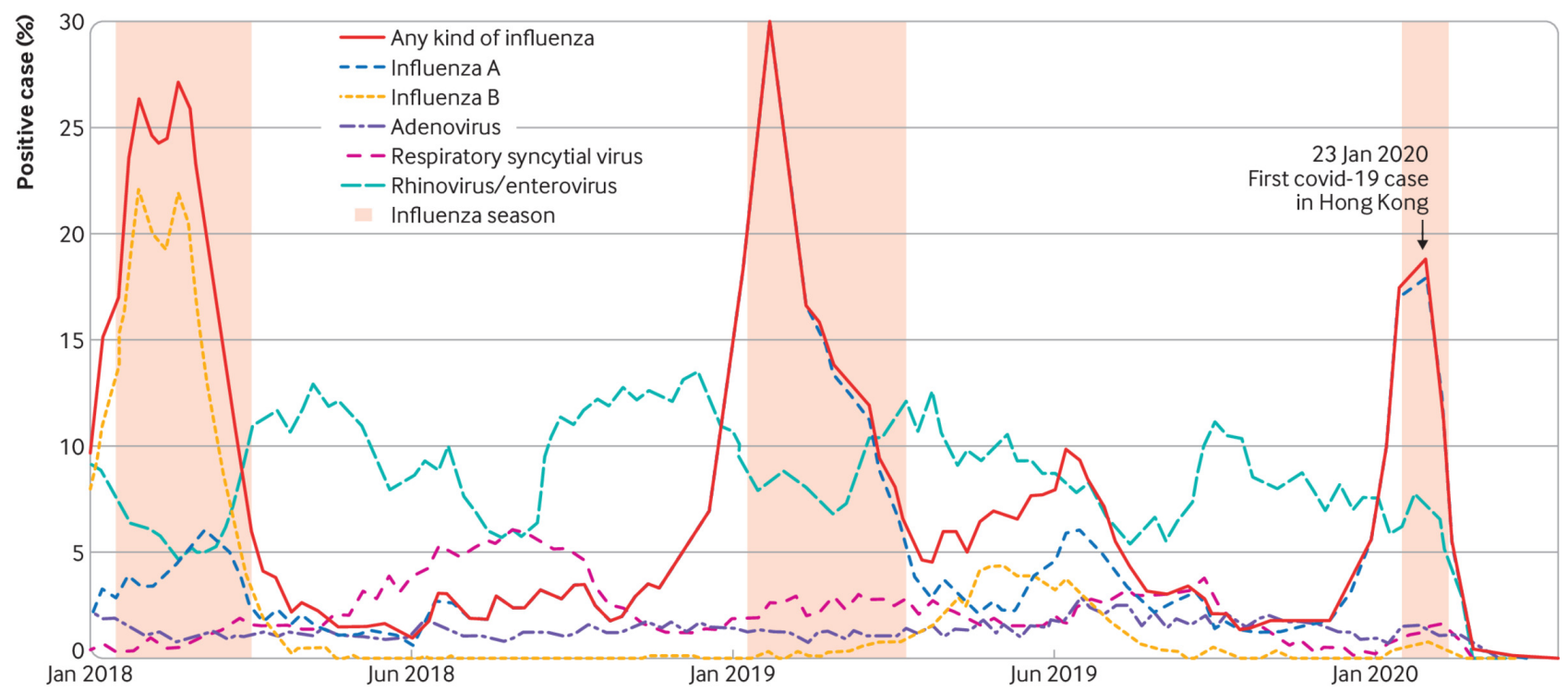

Fig 2 Time series of percentages of different pathogens in all respiratory specimens analysed in 2018-20 in Hong Kong. 


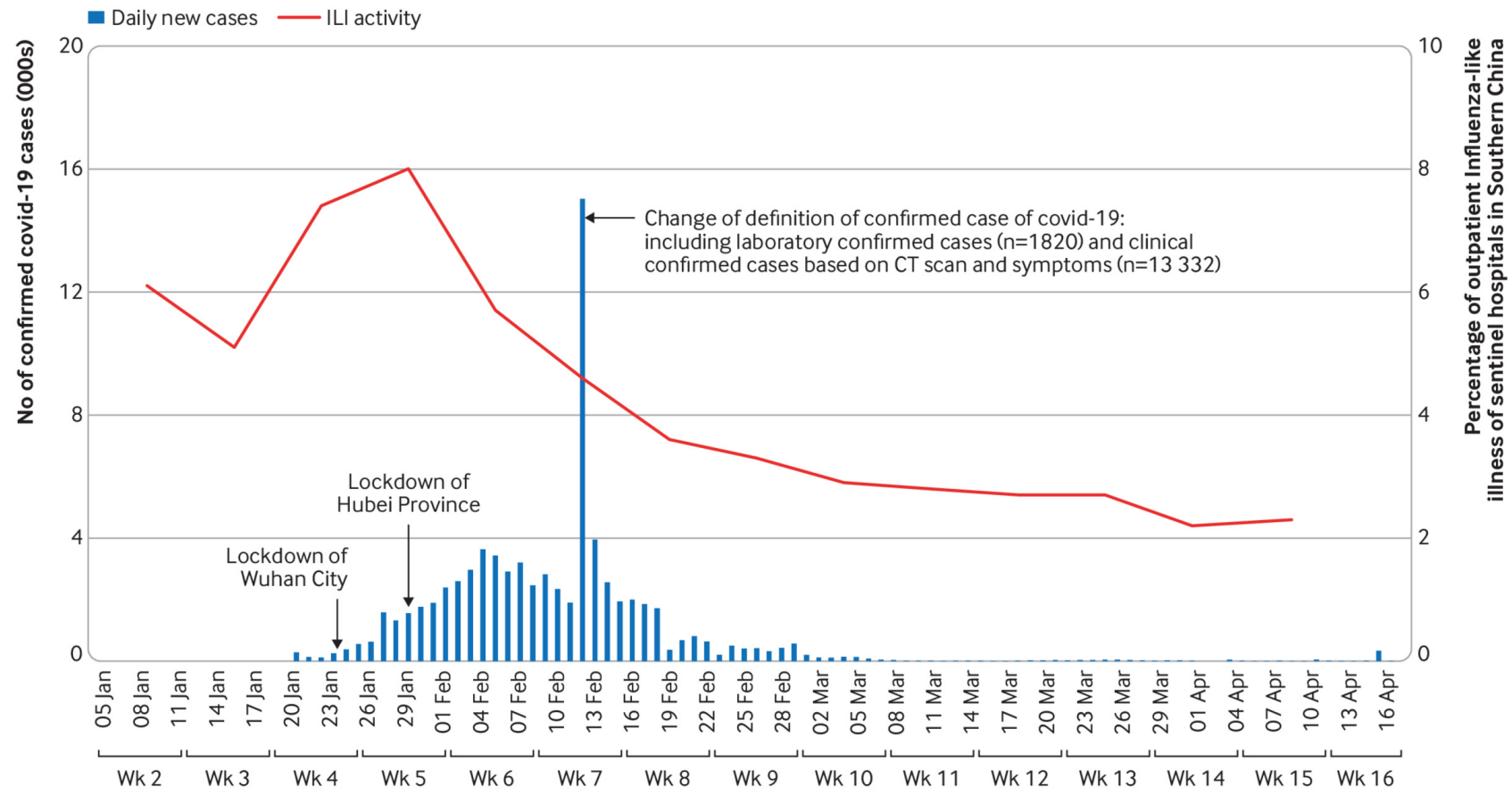

Fig 3 Incidence of covid-19 and indicators of transmission intensity of influenza-like illness from week 2 to 16 of 2020 in Southern China (Hubei, Shanghai, Guangdong, Jiangsu, Zhejiang, Anhui, Fujian, Jiangxi, Hunan, Guangxi, Hainan, Chongqing, Sichuan, Guizhou, and Yunnan). Lockdown of Wuhan City and Hubei Province involved shutting down all transportations in and out of the region and either restricting or banning local travel. Data source: Chinese National Influenza Centre. ${ }^{13}$ The latest data available at the time of writing were dated 17 April.

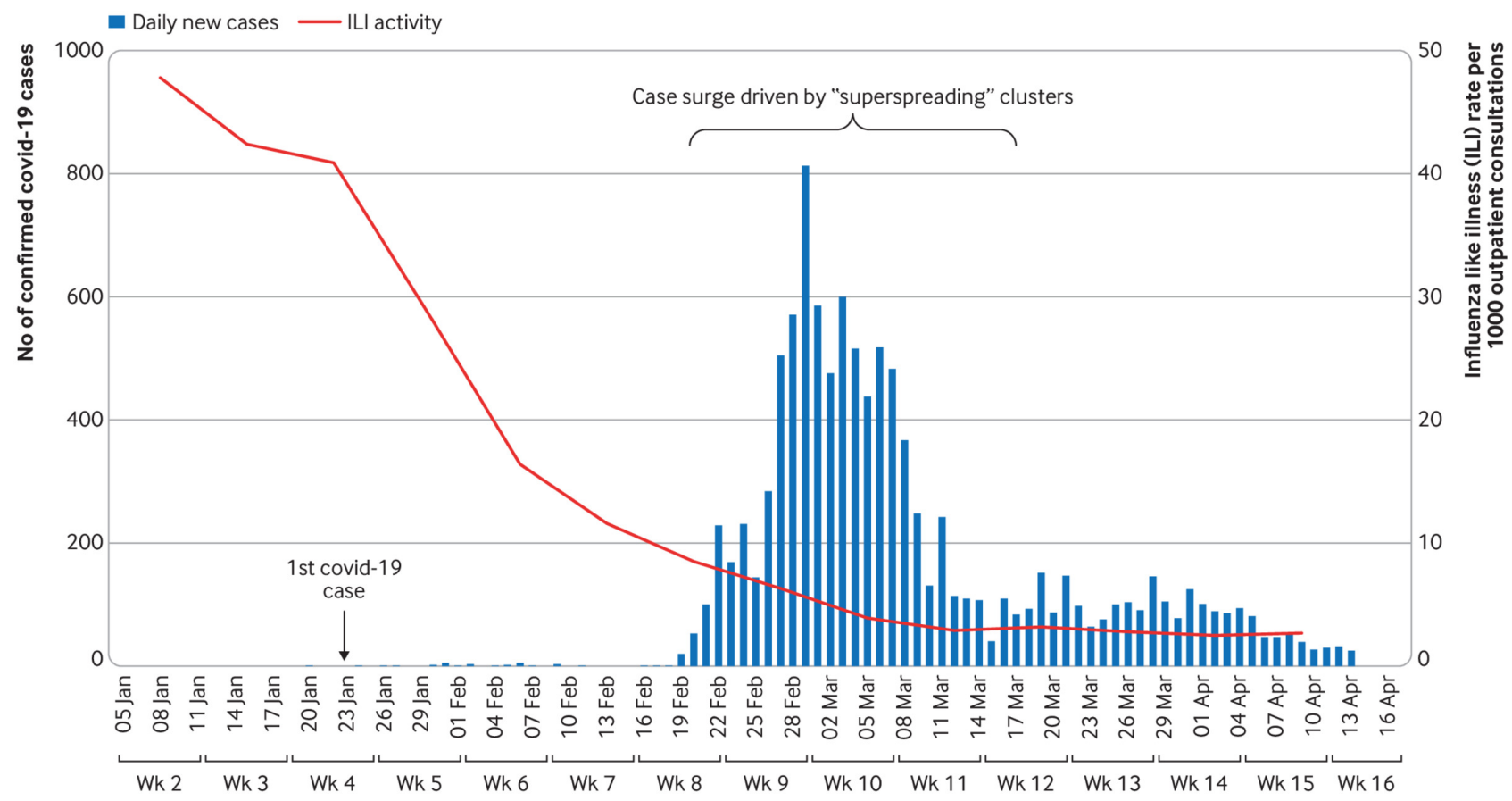

Fig 4 Incidence of covid-19 and indicators of transmission intensity of influenza-like illness from week 2 to 16 of 2020 in South Korea. Data source: Korea Center for Disease Control and Prevention. ${ }^{14}$ The drastic case surge in weeks 8-10 was primarily driven by "superspreading" clusters, including the Shincheonji Church cluster, in which 5016 members were infected $\left(60 \%\right.$ of all cases in South Korea) as at 18 March. ${ }^{17}$ The latest data available at the time of writing were dated 13 April. 


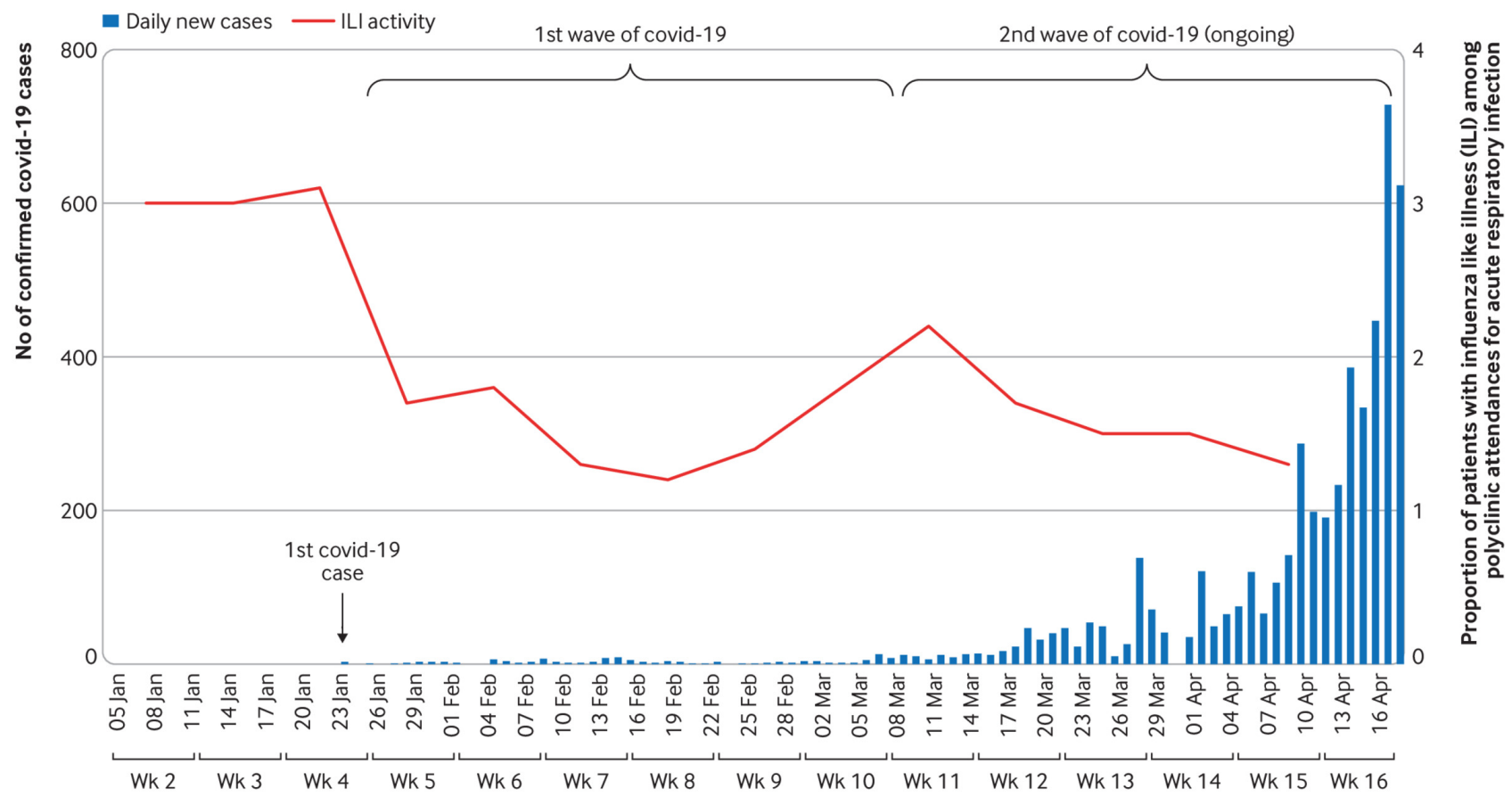

Fig 5 Incidence of covid-19 and indicators of transmission intensity of influenza-like illness from week 2 to 16 of 2020 in Singapore. Data source: Singapore Ministry of Health..$^{15}$ The latest data available at the time of writing were dated 18 April.

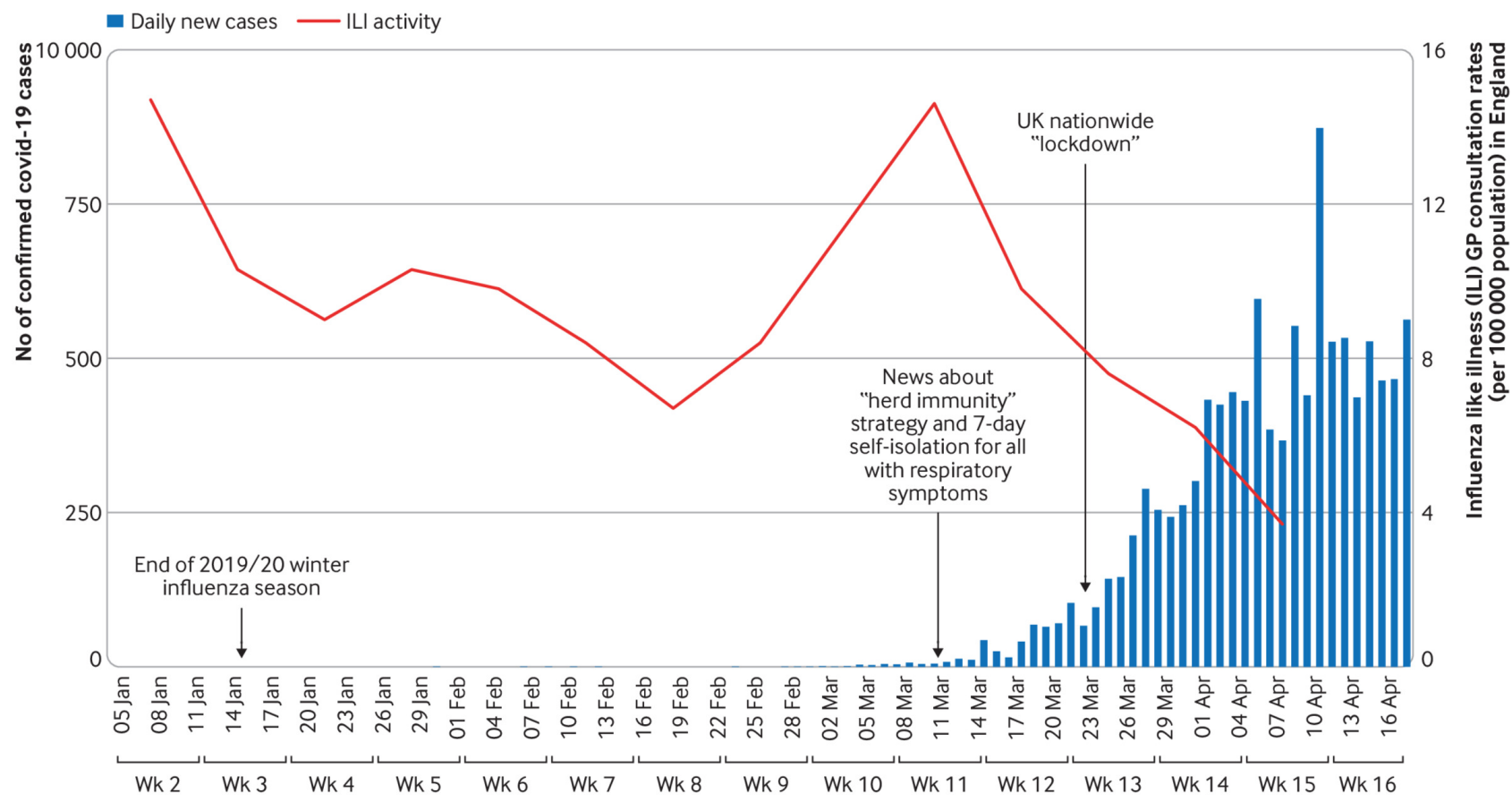

Fig 6 Incidence of covid-19 and indicators of transmission intensity of influenza-like illness from week 2 to 16 of 2020 in England. The 2019-20 winter influenza season started in week 49 of 2019 and ended in week 3 of 2020. UK nationwide lockdown involved shutting all non-essential shops (except supermarkets, pharmacies, newsagents, vets, petrol stations, hardware shops, banks, laundrettes, and undertakers), banning gatherings of more than two people except for households, mandating all residents to stay at home unless for essential travel (shopping for food or medicine, one type of exercise a day, medical appointments, as a carer visiting a vulnerable person, for essential work). Data source: Public Health England. ${ }^{16}$ The latest data available at the time of writing were dated 18 April. 